\title{
La Influencia del Derecho Francés en las Instituciones Jurídicas del Tercer-Mundo*
}

\section{The influence of French Law in the Legal Institutions of the Third World}

\author{
JeAn du BoIs de Gaudusson**
}

Fecha de recepción: 2 de marzo de 2009

Fecha de aprobación: 4 de mayo de 2009

\section{EN PRIMER LUGAR ALGUNAS PRECAUCIONES QUE NO SON DE ESTILO}

El tema que se nos ha solicitado tratar es delicado: nunca es fácil disertar sobre la influencia de su propio sistema jurídico sin correr el riesgo de caer en "el patriotismo jurídico" -familiar a muchos juristas franceses desde hace varios siglos- o de ser tratado de pesimista o... traidor si nos dejamos llevar por la crítica y la comprobación de la decadencia de la influencia jurídica francesa. La adopción -o los esfuerzos para ello- de una postura científica y de evaluación lo más objetiva posible, debería permitirnos evitar caer en esos dos obstáculos y medir, a la vez, la amplitud, las modalidades y el futuro de esta influencia del derecho francés en el Tercer Mundo, sin embargo, con un límite que tiene que ver con un determinado cargo en el derecho público que es nuestra especialidad disciplinaria... nuestras observaciones, que son las de un profesor de Derecho Público, se juzgarán quizás menos pertinentes en Derecho Privado.

La presente contribución no obedece totalmente a los cánones habituales de presentaciones académicas. Habida cuenta del tema del coloquio, del tema tratado y de la oportunidad que se nos da en este encuentro con intelectuales y universitarios, quienes pertenecen a otra cultura -aunque los puntos de aproximación con la nuestra son antiguos, numerosos y conocidos de ustedes- nosotros organizaremos nuestras proposiciones en forma de cuestionamientos, de interrogaciones y a veces de propuestas que podrán parecer provocadoras y quizás cuestionables; unos y otras sólo tienen por objeto suscitar un diálogo que no excluye ni debates, e incluso, controversias o críticas.

** $\quad$ Profesor de la Universidad de Montesquieu-Bordeaux IV (Fra.), expresidente de la misma universidad y directo del Centro de Investigaciones comparadas en Derecho Constitucional, Administrativo y Político. El presente trabajo constituye la versión escrita de la intervención del profesor du Bois de Gaudussón en el seminario La influencia del derecho francés en el derecho colombiano, celebrado en octubre de 2007 en la Facultad de Derecho de la Universidad Santo Tomás. 
Los términos del tema, ameritan varias observaciones que nos parecen necesarias para evitar los malentendidos, las ambigüedades o incluso los pleitos de intención, más necesarios, en cuanto que se cuestionan aquí fenómenos que no son puramente jurídicos o, que si lo son, no se incluyen sino dentro de su contexto político e ideológico.

\section{La influencia}

Las influencias entre sistemas jurídicos son una realidad, la cual, lejos de ser nueva, actualmente, conoce un desarrollo considerable: se observa en la época contemporánea la multiplicación de fenómenos calificados de transferencia de tecnología, migración, imitación, transplante... en el mundo; se les estudia cada vez más y son valorizados : si hay un nuevo tema en el mundo científico de los juristas, es el del comparatismo y el empleo de argumentos de Derecho Comparado. Las ramas del Derecho y de los sistemas jurídicos de los Estados están bajo influencia y cada vez más juristas sacrifican el comparatismo con más o menos éxito, pero, generalmente, por necesidad.

Si no es nuevo, este fenómeno cambia de aspectos; reviste formas variadas que aparecen de manera más o menos libre, consentida o forzada, pacíficas o violentas. Su objeto es en sí mismo variable cuando se analiza en la implantación de un sistema jurídico en su totalidad, o en la adopción o préstamo de una técnica, de una regla, de una institución, de un mecanismo. En otros casos, que nos conciernen aquí, se tratará la influencia de referencias a fuentes materiales del Derecho a la promulgación de normas, las cuales se inspiran, por distintas razones en un sistema jurídico que tendrá los caracteres de un modelo jurídico. Al respecto, e indiscutiblemente, el derecho francés tiene la reputación de ser un modelo y es fuente precisamente de influencias. Sin embargo, los interrogantes se plantean a propósito de saber en qué medida y según qué modalidades esta influencia se ha ejercido, sobre todo, en el pasado.
Se observará que no siempre es fácil analizar las influencias de los sistemas jurídicos, cuando ellas son parasitadas por la utilización de conceptos con fuerte connotación política, por enfoques sumarios o incluso caricaturales, tributarios de relaciones de fuerzas internacionales y políticas, obteniendo como consecuencia la transformación de la realidad estudiada. Ya volveremos sobre este tema.

\section{El derecho francés}

La expresión en sí misma no suscita debate. No obstante, cuando se trata de determinar su influencia, una serie de interrogantes se plantean: ¿de qué derecho se trata? ¿Del derecho del siglo XXI o del derecho de siglos anteriores? Incluso, la cuestión es más pertinente cuando se observa en el ámbito jurídico esa famosa aceleración, que, según Daniel Halévy, caracteriza la historia contemporánea. No se cuentan ya las reformas legislativas ni los cambios jurisprudenciales, ni los nuevos debates doctrinales: ya al final del siglo XIX, con respecto a la decisión del Tribunal de conflictos en "Canal de Gignac", Maurice Hauriou exclamaba "nos cambian nuestro Estado". Ésta expresión, aplicada al Derecho Administrativo, es regularmente utilizada, sin duda de manera excesiva, pero que en todo caso designa una realidad cuando numerosas disciplinas del derecho francés se transformaron. Sin embargo, nos queda decir que si numerosos sectores y técnicas jurídicas cambian, esas evoluciones no significan necesariamente la convulsión, ni el cuestionamiento de todos los grandes equilibrios ni el abandono de los principios fundadores, ni aún menos, el rechazo de lo que constituye la esencia del derecho francés $y$, sobre todo, su pertenencia a la escuela romano-germánica.

Simplemente, por el momento, tengamos en cuenta que las conclusiones de los estudios hechos sobre la influencia del derecho francés variarán según se retenga una técnica, un sector, reglas generales, un principio o incluso una corriente 
doctrinal, una tradición jurídica... Agreguemos que la intensidad de la influencia no se mide idénticamente y que su realidad varía según las ramas del derecho. Allí hay un campo muy extenso de trabajos a los cuales se dedican numerosos investigadores colombianos.

Conviene insistir en una dificultad suplementaria que se refiere a la existencia y a la identidad misma de un modelo jurídico francés: ¿cuál es ese modelo en una época en el que se multiplican los intercambios entre sistemas jurídicos, los procesos de hibridación y el mestizaje jurídico, así como las imitaciones crecientes de normas extranjeras a las cuales se libra el Derecho francés? La europeización del Derecho, su internacionalización, o incluso su globalización no dejan mucho espacio a un derecho que sería puramente nacional y sobre el cual, el Estado y sus relaciones tendrían una determinada influencia. Por último, este modelo, suponiendo que se llegue a identificarlo, ino ve su territorio estrecharse? El "Derecho nacional" no constituye en el día de hoy más que una porción reducida del Derecho aplicado; en adelante, se inserta en una red de normas de toda clase, procedente de nuevos centros de poder y de producción del Derecho, que se imponen a los ciudadanos y a los juristas franceses.

Estas observaciones se refieren al conjunto de los sistemas jurídicos y también pueden aplicarse al derecho español, alemán o anglosajón; pero éstas revisten de una fuerza particular y tienen una significación altamente simbólica para el derecho francés tradicionalmente considerado como un producto de exportación y a propósito de una doctrina jurídica francesa cuyos análisis, reflexiones y teorías también ejercen tradicionalmente una innegable influencia. Allí hay un dato para tener en cuenta en nuestra reflexión.

Finalmente, tenemos el Tercer mundo. La introducción de este dato en nuestro debate dista mucho de ser neutro. Dentro de su complejidad, éste es de una naturaleza tal que puede ayudarnos entender mejor los distintos aspectos de los problemas de la influencia de un sistema jurídico. No nos ocuparemos del origen del de la expresión “Tercer mundo", ni sobre las variaciones de su consistencia y las dificultades de definir las fronteras, ni sobre sus nuevos desarrollos. Categoría heterogénea si no heteróclita, que hoy en día efectúa hoy -bajo otros vocablos- su regreso a la escena internacional, en una época en la cual la mundialización revive tensiones y conflictos. Un elemento de su unidad reside en la relación que ella mantiene con las potencias del Norte y en su menor desarrollo, aunque desde ésta óptica convenga operar distinciones dentro de lo que se llama el "Sur", convirtiendo en gran medida caduca la tradicional oposición Norte-sur...

En cualquier caso, a primera vista no está garantizado que este concepto logre efectuar una especificidad de la influencia del derecho francés sobre los países del Tercer Mundo, distinta de la ejercida sobre el resto del mundo, salvo dos reservas sobre las cuales quisiéramos insistir, ya que son esenciales para nuestro tema.

En primer lugar, es evidente que la influencia del derecho francés no es exactamente la misma según las historias jurídicas (y obviamente políticas) de países, dependiendo de si estos países han sido o no colonizados por Francia. Esta influencia se ejerció de una manera muy particular con respecto a los países del Tercer Mundo que se encontraron bajo la soberanía política de Francia, como fue el caso, por ejemplo, en África. Es interesante examinar la situación de los primeros, por una parte. Así como para las otras dominaciones coloniales, ésta es la causa de los análisis de los fenómenos de transferencias y flujos jurídicos cuestionables, a menudo demasiado sumarios y caricaturales. Por otro lado, dicha situación se transforma hasta acercar a estos países, progresivamente, a los otros países del Tercer Mundo, e incluso, a los otros países del mundo, haciéndolos participar, a su vez, en 
este famoso "mercado internacional del Derecho" que se construye en el día de hoy.

En segundo lugar, cualquiera que sean sus diferencias, los países del Tercer Mundo se encuentran, por múltiples razones, enfrentados más que otros a elecciones de sistemas, de técnicas jurídicas, en el marco de la construcción de su marco jurídico e institucional: frente a estos países, el derecho francés -en competencia con otros- se encuentra enfrentado al reto de su propio poder de atracción no sólo para permitirle seguir ejerciendo su influencia en un mundo globalizado, sino también para responder a las expectativas propias de los países del Tercer Mundo.

\section{LA INFLUENCIA DEL DERECHO FRANCÉS EN LOS PAÍSES DEL TERCER MUNDO DE HERENCIA FRANCESA}

Si hay una práctica bien afianzada en la literatura jurídica y constitucional sobre los países de herencia francesa, comenzando, sobre todo, por los Estados africanos que son considerados aquí, es la que busca dar cuenta de los regímenes constitucionales y sistemas jurídicos, en general, en términos de mimetismo. Este enfoque es tentador y justificado para caracterizar regímenes marcados durante mucho tiempo por su relación de dependencia y pertenencia a un mundo cerrado sobre el exterior, teniendo en cuenta también las fuertes semejanzas y múltiples similitudes observadas entre los derechos africanos y los derechos exteriores, como el derecho francés que los influencia plenamente.

El isomorfismo constitucional y los acercamientos son tales que terminan por transformar la operación de promulgación de normas nacionales en un ejercicio de "calcomanía jurídica e institucional", retomando la expresión del juez Bedjaoui. Sobran ejemplos de dichas situaciones. A menudo, incluso éstas se han acentuado en el momento de la descolonización: los países conocieron y se encontraron durante el periodo colonial bajo la influencia del derecho francés, pero se trataba de un derecho endulzado, en numerosos aspectos diferente del derecho de la metrópolis (véase, por ejemplo, el principio de especialidad legislativa). Desde su independencia política, adquirida a principios de los años sesenta, estos países no sólo heredaron el modelo jurídico francés, sino que también, a menudo, se han acercado hasta el punto de alinear su corpus jurídico al módulo francés (ejemplo es el del derecho de la función pública). De la misma forma, y por otras razones, el texto de las Constituciones africanas elaboradas en los años noventa es ciertamente mucho más cercano al constitucionalismo francés de lo que era en los años setenta u ochenta.

Apreciación crítica del enfoque mimético: que se nos perdone consagrar un desarrollo que podría parecer redundante a algunos sobre esta tentación de numerosos investigadores franceses de focalizar la problemática y el estudio de los sistemas jurídicos africanos sobre el mimetismo. Esta tendencia doctrinal, siendo especialmente clara en algunos sectores jurídicos, como en el Derecho Mercantil o el Derecho Público y particularmente en el derecho constitucional que se analizará específicamente para ilustrar nuestras observaciones. Estas son dos: en primer lugar, la referencia al mimetismo oculta ambigüedades y es fuente de confusión, de interferencia, poco apta para hacer un análisis científicamente pertinente de la realidad de los flujos y transferencias jurídicas y de las condiciones en las cuales opera el proceso de producción normativa. Existen dos efectos ópticos de los cuales conviene desconfiar. Nosotros tenemos en cuenta como apoyo de nuestra demostración un ejemplo, el del constitucionalismo:

Así, si el mimetismo se define como la adhesión de los constituyentes a los valores de la democracia liberal y pluralista, a la apropriación de un principio de organización de las autoridades públicas o también al propio constitucionalismo; algunos ven en esto la explicación de otra característica 
fundamental del Derecho Constitucional en África, es decir, su inadaptación. Ciertamente, los orígenes del constitucionalismo -como las del Estadonación- se sitúan fuera de África. ¿Pero se puede deducir que éstos valores e instituciones son por esencia y para siempre no solamente extranjeros para el África, sino, como algunos lo afirman, le son también inadecuados?

Una confusión común vincula principios políticos y filosóficos con regímenes constitucionales. Ahora bien, la historia muestra que a la identidad de principios no corresponde necesariamente a la identidad de la organización de las instituciones, pero está lejos de ser así. El debate sobre el mimetismo se reviste de una verdadera pertinencia, sólo si él se refiere a los regímenes constitucionales y sus técnicas.

Sobre este último punto, el análisis en términos miméticos encuentra también rápidamente sus límites. Primero, porque la realidad de los textos es otra. Ya volveremos sobre este punto. Segundo, debido a la dificultad de determinar la frontera entre aquello que concierne al préstamo y lo que no le concierne. ¿A partir de qué grado de similitud de las normas se puede hablar de mimetismo? ¿Dichas normas, en definitiva, son subalternas, cuando lo esencial reside en la influencia general que era ejercida en África por modelos, esquemas y expertos exteriores impuestos? Tantas preguntas manifiestan interferencias y ambigüedades.

En realidad, el debate se sitúa a menudo en otro campo: derribado por el relativismo. el mimetismo es la calificación menos precisa para una situación concreta con respecto al reproche que se hace al África de no haber sabido afrontar un reto, el de elaborar un modelo constitucional original, diferente a otros prototipos, que se adaptaría a los valores culturales y a las especificidades de la sociedad africana. Se puede dudar de la adecuación de una serie de disposiciones a un determinado contexto cultural o social y sobre las posibilidades de aplicación de las disposiciones tomadas pura y simplemente de otro lugar. Nos queda preguntarnos legítimamente sobre el contenido de esos valores socioculturales, los cuales se espera que sean explicados. Cualquiera que sea la parte de verdad, en todo esto, la crítica presupone, como ya lo hemos recordado, en el fondo, la inadecuación del principio de las reglas de derecho constitucional en vigor por fuera de África. Ésta participa, también, en ésta tendencia a proyectar sobre ella misma esos modelos ideales y utópicos hechos de democracia directa, de popularización general del poder, cuya aplicación se espera siempre por fuera incluso de África... No insistiremos en un proceso que demuestra que se informó sin verdadera prueba... y que, si existe un fundamento, éste no pertenece a los Estados africanos como lo demuestra la historia de las constituciones y del constitucionalismo en el mundo.

Otra ambigüedad del análisis se origina en la impresión, según la cual, el mimetismo sería una característica, fundamental y específica del Tercer Mundo... Ahora bien, cada cual lo sabe, y Jean Rivero lo mostró en términos magistrales en las páginas consagradas a Ganshof Van Der Meersch en 1972, los fenómenos de imitación marcan y siempre han marcado la creación de los sistemas jurídicos. Según la palabra de Pericles, raros son los regímenes políticos que "no se propusieron como modelos las leyes de otros".

Esta utilización del análisis de los sistemas jurídicos del Tercer Mundo en términos de mimetismo da una visión que empobrece la producción normativa de esos países.

Existe un discurso que reduce la promulgación de las normas jurídicas -tomaremos aún el ejemplo del Derecho Constitucional- en África a no ser más que el producto de una influencia general y omnipresente de modelos y concepciones forjados bajo otros cielos. Última fase del razonamiento mimético, el derecho constitucional en África resultaría de un 
simple ejercicio de copia realizado bajo la autoridad de proveedores de fondos internacionales y extranjeros y bajo la potestad de sus hombres de pluma y ciencia jurídica. Se ve allí un factor suplementario de ineficiencia de un corpus de contenido, juzgado desde el principio como inadecuado, debido a sus condiciones de adopción: el espiral del mimetismo y de la ineficiencia se engancha y se acelera con una consecuencia principal y es de alimentar esta corriente doctrinal y política sistemáticamente crítica del derecho en África y de contribuir, así, a su reducción y a su devaluación; en África y también en los otros países del Tercer Mundo, víctimas de esta nueva dependencia jurídica.

Esta presentación no es sin fundamento y no se carece de pertinencia y desde hace casi 50 años, los ejemplos no faltan. Precisamente, en su límite, tal análisis no es de aplicación general y sobre todo no nos parece dar cuenta de las recientes evoluciones, ensayadas en África desde 1990.

La realidad es en efecto más compleja. En primer lugar, las nuevas constituciones son el resultado de un verdadero proceso de elaboración y creación normativa. Aquí, como en otros ámbitos y sin importar las semejanzas, la producción del derecho en África se hace... en África con los africanos y no solamente con otros. Hay, para reanudar la observación de G. Balandier, una articulación "de las dinámicas del interior y del exterior"; las segundas existen; las primeras también fueron seguramente escasas, pero se desarrollaron y en el periodo contemporáneo, la comprensión de las nuevas disposiciones constitucionales, de su naturaleza y de su significado pasa por un estudio de las modalidades de su elaboración y del papel desempeñado por los protagonistas políticos y sociales. Sin duda, la observación no es tenida en cuenta a priori, puesto que deja entrever que el Derecho Constitucional, en su nueva evolución no es simplemente un artículo de importación y que él no se revela simplemente como un orden simbólico o de encantamiento.
Estudios, aún demasiado raros, comienzan a demostrar que las constituciones de los años noventa fueron redactadas de manera menos unilateral que aquéllas anteriores a los años sesenta, situación que no se menciona.

Las constituciones se elaboraron según procedimientos que hacían intervenir varios órganos, más o menos formalizados, más o menos inéditos. Al respecto, es necesario citar obviamente las conferencias nacionales, esos "hallazgos políticos del África poscolonial"; son una "invención propiamente africana" retomando las expresiones del profesor Maurice Kamto, y no se pueden considerar como simples mezclas de experiencias históricas francesas o norte-americanas, como muchas veces se ha escrito... Además, la elaboración de las constituciones se efectuó bajo normas de excepción y en distintos grados, dando ocasión a discusiones y debates entre tesis divergentes, a versiones sucesivas, a transacciones y a arbitrajes. A veces, esta dimensión es minimizada por el contenido de los textos en los cuales se materializaron estos procedimientos.

Pero, sobre este punto también se imponen matices y precisiones. Ya se mencionó que más allá de las semejanzas, los regímenes constitucionales varían de un Estado a otro; basta con comparar las Constituciones de Madagascar, de agosto de 1992, de Benín, de diciembre de 1990 y de Malí, de agosto de 1991, para convencerse de la imposibilidad de reducir a la unidad las constituciones africanas. La influencia de modelos exteriores no es discutible, y los constitucionalistas encontrarán sin dolor la reproducción idéntica -o casi- de capítulos o artículos enteros de la Constitución francesa. Es el caso frecuente con respecto a la organización del Parlamento y a la definición del ámbito de la ley, las modalidades del control jurisdiccional del poder, o también el estatuto del Presidente de la República. Pero estas aproximaciones no permiten ver en estos textos simples "constituciones-eco". 
En toda hipótesis, la interpretación del isomorfismo constitucional y jurídico no se puede hacer sin algunas precauciones. La reproducción de disposiciones y artículos está cargada de significados, habida cuenta del momento histórico en el cual se sitúa la operación constitutiva: la vuelta al constitutionnalismo liberal y a un texto más próximo al de Francia, presente en varios países, es la manifestación de una ruptura con el orden constitucional previo; muchas normas y mecanismos, incluso muchos de ellos inspirados en el extranjero, aparecen como innovaciones y se viven como tales; son la marca de otra ruptura, ésta vez política. Buena cantidad de las disposiciones fueron objeto de debates muy vivos en el momento de su adopción. Por ejemplo, pensamos en los pasajes relativos al Gobierno y a las relaciones del ejecutivo con el Parlamento.

También se recordará que los mismos regímenes constitucionales se pueden reutilizar, diremos reapropiar en función de un contexto, de circunstancias, de relaciones sociopolíticas, distintas a aquéllas que presidieron su definición inicial. El transplante de modelos jurídicos se acompaña siempre de un fenómeno de reapropiación. Una vez importada la institución, ésta funciona en el contexto del sistema de valores y significados de la sociedad receptora. Hay toda una serie de estrategias de reinvención o incluso de "canibalización" o, en cualquier caso, una aclimatación de la institución que modifica su alcance y su significado inicial. De igual forma, la historia nos enseña que pueden existir varias "lecturas", es decir, interpretaciones y aplicaciones de una misma Constitución. En África, como en otras partes del Tercer Mundo, el comentario de las constituciones pasa, incluso, por parte de los juristas, por la consideración del contexto político y social. Es una evidencia que no es inútil recordar a la escuela de la exégesis.

Estas observaciones tienen un alcance que supera el derecho constitucional. En otros sectores jurídicos, el enfoque mimético es especialmente inadecuado, tanto que el derecho llega a poseer un contenido diferente del de la antigua metrópolis (véase el derecho de familia, derecho de los bienes por ejemplo).

\section{LA INFLUENCIA DEL DERECHO FRANCÉS EN LOS PAÍSES DEL TERCER MUNDO EN UN NUEVO CONTEXTO}

Los países del Tercer Mundo conocen evoluciones que transforman las condiciones de construcción de sus sistemas jurídicos, lo cual implica consecuencias sobre la influencia del derecho francés. El caso de los países que soportaron la soberanía política de Francia es al respecto especialmente típico.

$1^{\circ}$ Los sistemas jurídicos de estos países se abrieron progresivamente a otras influencias. Se asiste a lo que se podría llamar una "desmonopolización del pabellón". Los Estados en cuestión, como otros, se beneficiaron de las posibilidades de elección que los ha convertido en más libres en la construcción de sus sistemas jurídicos. Ellos se presentan, como otros, en un mercado internacional del derecho que se estableció gracias a la diversidad de sus protagonistas, tanto del Norte como del Sur (existen transferencias Sur-Sur). En éste mercado cada uno hace su "almacenaje", no siempre con plena racionalidad; las transferencias (compras) no se refieren más a los códigos -y aún menos a los sistemas- llave en mano, completos, sino más bien a los repuestos: eso sin plantear delicados problemas de compatibilidad e integración en el sistema existente. La importancia de este nuevo mercado (forum shopping) no se puede minimizar tanto para la adopción de un "repuesto" que puede modificar profundamente Ios equilibrios jurídicos previos y otros. Ustedes saben mejor que otros el significado y el alcance de la creación de un Consejo de Estado inspirado del derecho francés.

El desarrollo de éste "comercio" jurídico e institucional, en parte organizado por protagonistas 
influyentes (instituciones internacionales, proveedores de fondos, "modelos históricos") contribuye a una convergencia en una serie de ámbitos. Esta convergencia no es, conviene observarlo, solamente la expresión de un fenómeno de soberanía cultural. También es el resultado de una estrategia deliberada de las elites locales, a fin de sacar provechos simbólicos y prácticos (véase en este sentido los trabajos realizados en la Universidad Montesquieu de Bordeaux, por ejemplo, los de Dominique Darbon, Sory Baldé y otros).

$2^{\circ}$ Este análisis del modelo jurídico, en términos de competencia, no debe ocultar el hecho de que para muchos países del Tercer Mundo, el derecho francés sigue siendo una referencia y conserva su influencia de varias maneras.

En primer lugar, el derecho francés ejerce su influencia en países que comparten el mismo patrimonio jurídico. Sea cual fuere la historia y las modalidades de las transferencias jurídicas, se constituyeron en el mundo conjuntos jurídicos, escuelas de derecho que reunieron países con experiencias diferentes. Es el caso de la pertenencia a la tradición romano-germánica. Así se explica lo que se llama, como ya se dijo de manera inadecuada, "mimetismo", pero que participa también de "ese efecto de familiaridad", que tiene la pertenencia de los Estados en cuestión a una misma escuela, formada por conceptos, principios y vocabularios comunes. Para reanudar el ejemplo del derecho constitucional, cualquiera que sea la fuerza de la uniformación constitucional contemporánea en el mundo, existen "escrituras de la Constitución" propias a grupos de países que hacen que las cuestiones constitucionales se aborden de manera idéntica o muy aproximada: las Constituciones se redactan según un mismo tipo de plan, se definen las competencias según una misma rejilla de distribución, de instituciones comunes que se encuentran sistemáticamente. La lectura de los textos constitucionales muestra evidentemente la existencia de una escuela francesa, como existe una escuela portuguesa (como lo prueba la comparación de las constituciones portuguesa y angoleña) o americana o también alemana... Entre los Estados de sucesión francesa hay una semejanza formal -que dista mucho de ser desdeñable- pero también conceptual y sustancial; esto último puede ser más o menos evidente; hasta se puede haber convertido en muy escasa, sin que por ello cambien las presentaciones, las palabras y los conceptos. ¿Qué decir también del código civil, ejemplo espectacular de ésta influencia?

Se intentaría, sin querer justificar las relaciones de fuerza... hablar de una influencia de un derecho francés compartido y constatar que existe una misma escuela jurídica organizada en torno a un "patrimonio jurídico común", sin que por ello se pueda minimizar los efectos sobre la soberanía y las condiciones de violencia (colonial, económica) dentro de las cuales se construyó esta escuela. Pertenencia que de forma amplia da origen a numerosas "versiones" jurídicas.

En segundo lugar, porque el derecho francés ejerce una atracción comparativa en el mundo. Ésa es al menos la hipótesis sobre la cual sugerimos reflexionar.

El desarrollo del mercado internacional del derecho es abastecido por el desarrollo del derecho comparado que ofrece a todos la existencia, real, o supuesta, de otras soluciones, de otros modelos. Es época de evaluación y de comparaciones, como lo prueba de manera brillante el éxito del enfoque económico del derecho, utilizado con el objetivo de enfrentar las ventajas, los inconvenientes y los costos de los sistemas jurídicos y sus técnicas. La evaluación económica comparativa del derecho se volvió central, tanto para los investigadores como para las instituciones multilaterales; está en el centro de la competencia abierta por la mundialización (o globalización), abastecida por los informes del Banco Mundial (Doing business), entre dos sistemas jurídicos y una voluntad de crear un derecho favorable a la inversión, superando el 
"common law" y el derecho Romano-germánico. Es en tal contexto que cada vez más el derecho francés, como otros, se somete a la evaluación de su eficacia y de su poder de atracción. (Sobre estas cuestiones, véase, en particular, los trabajos de J.B Auby, de B. Du Marais coordinador de un Programa Internacional consagrado "a la atractividad económica del derecho" en el cual presidimos el Consejo científico, con tres obras que aparecen en la Documentación francesa; véase también el estudio del Consejo de Estado francés sobre "la influencia internacional del derecho francés", 2001".

Esta situación es nueva: después de haber ejercido en la historia considerables influencias como lo prueba la difusión del código civil, el impacto del derecho administrativo, la contribución principal del derecho francés en la construcción del Derecho comunitario europeo, el sistema jurídico francés no se encuentra marginalizado y se plantea la cuestión de saber si es aún deseable (Christophe Jamin). No se trata tanto de saber si tal o cual técnica del derecho francés es mejor más exitosa que otras pertenecientes a otros sistemas -los ejemplos al respecto son numerosos-, sino de preguntarse sobre las especificidades del propio sistema y determinar si éste está en condiciones de enfrentar la competencia internacional y, en particular, las tradiciones del "common law". Con una pregunta subsidiaria, y es la de saber si el derecho francés, mientras permanezca identificable, aporta respuestas adaptadas a las expectativas del Tercer mundo y que no aportarían otros sistemas jurídicos.

Sin subestimar las dificultades de tal investigación y los riesgos de sobrevalorar su propio sistema, es posible dilucidar varias características que nosotros sometemos a valoración crítica.

Hay en primer lugar una "atracción epistemológica" con lo que ello significa, esto es, de la manera específica de pensar el derecho.
Para los autores, cuyas conclusiones retomaremos, de una reciente obra publicada por la Asociación Henri Capitant sobre los derechos de tradición civilista, preocupada por aportar respuestas a los informes Doing business, el derecho francés se beneficiaría de una serie de "activos estructurales":

- La accesibilidad: gracias, en particular, a la codificación, método que ha sido tenido en cuenta por los propios expertos americanos para participar en la creación del nuevo derecho económico de países del Este europeo y que también es utilizado en China, en Vietnam y lo fue en Japón a partir de la era Meiji. El acceso material, es decir, la posibilidad de conocer la regla, es más fácil cuando ella es objeto de un texto de ley, que recurrir a múltiples decisiones jurisprudenciales. En efecto, el common law es, a menudo, accesible sólo para los iniciados, pues no es fácil para los ciudadanos poder dilucidar una regla de múltiples jurisprudencias, suponiendo que puedan acceder a ellas, lo cual en la práctica es imposible para la mayoría. Se trata entonces de un "modelo poco exportable". El acceso intelectual resulta de la intelegibilidad de la regla. Si el Código civil francés tiene tanto prestigio mundial, es porque está escrito en un lenguaje "popular" en el sentido de que todo ciudadano lo comprende. ¿ No podríamos decir entonces que los derechos romanistas son más "democráticos"?

A estos argumentos conocidos, se añadirá esa pasión francesa por la forma, a tal punto de confundirse con ella. La mejor expresión de ésta reside en el espíritu codificador; del Código civil a la codificación del resto del derecho, tanto en el Derecho Privado como en Derecho Público. No olvidemos que el Consejo de Estado francés desempeñó en Derecho Público el mismo papel que el Código Civil en Derecho Privado: permitió poner en orden y sistematizar el derecho en su conjunto: uno y otro han permitido poner 
en orden y sistematizar el derecho, dar coherencia a un sistema globalizante, proyectando a nivel jurídico un verdadero proyecto político. Sin embargo, la pregunta se plantea en saber si esa coherencia hoy en día no es puesta en entredicho por la "lógica de las consecuencias" en pleno desarrollo, que obliga a interesarse mucho más en las consecuencias de las decisiones y en razonar en los términos del law and economics.

- La seguridad: debido a que la solución es dada por la ley con antelación a todo contencioso, el derecho romanista asegura una prevención de litigios. Un pleito y la intervención del juez son fracasos del Derecho y no las señales de su triunfo, como lo hacen creer los propagandistas del common law. La seguridad, aunque genera un costo, tiene un valor económico, incluso, superior a la falsa economía del más pequeño y aparente costo inmediato.

- La flexibilidad: ya que contrariamente con las apariencias, la norma jurisprudencial del precedente retrasa las adaptaciones que la ley puede procurar instantáneamente. Una buena norma codificada también se puede prestar fácilmente a interpretaciones jurisprudenciales que le abrirán a veces ámbitos de aplicación extensos y diversificados, como es el caso de los artículos 1134 (contratos) o 1382 (responsabilidad) del Código civil.

Existe a continuación una atracción sustancial.

A menudo, se destacan los "activos sustanciales" del derecho francés y de manera más general de los derechos romanistas, tomando el ejemplo del derecho de los contratos: es un derecho abierto, debido a que es más fácil modificar una ley que transformar una jurisprudencia, incluso, mediante la importación de soluciones extranjeras; un derecho equilibrado, lo que apoya un desarrollo de la libertad contractual y sus límites; un derecho económico que no tiene necesidad de recurrir, por una parte, a la impresionante letanía de definiciones y cláusulas de los contratos anglosajones, necesaria no por deseo de precisión sino por falta de referencias o para prever los casos que la ley no precisa, dando lugar, por otra parte, a innumerables pleitos para regular lo que la ley romanista habría regulado por adelantado. En total, el costo de los contenciosos y de la justicia en sentido más amplio en un país de common law propicio a los contenciosos como los Estados Unidos, representa un 2,6\% del producto nacional bruto contra un $0,6 \%$ en Francia; hay un abogado por cada 300 habitantes en los Estados Unidos contra uno por cada 1.700 en Francia; un adulto sobre 10 tiene relación con un pleito en los Estados Unidos contra uno sobre 300 en Francia.

Muchos otros ejemplos podrían ser citados. Para continuar con el sistema francés tomado en su globalidad, un factor de atracción resulta ser la tradición de apego a establecer un equilibrio -se le calificará como tal- entre dos exigencias contradictorias: la del interés general y la defensa de los derechos humanos. El tema es obviamente clásico, pero reviste una actualidad más viva con el acento que cada vez se pone más sobre los derechos subjetivos, los derechos de los individuos, a veces con tal agudeza cabe preguntarse sobre el futuro de la defensa del bien común. El derecho francés se caracteriza por esta evolución, constatada en los países posmodernos, pero su compromiso en pro del servicio público, por ejemplo, ofrece una de las vías para mantener la lógica esencial de un derecho orientado hacia el interés general. Los dos derechos subjetivos e interés general están (o deben estarlo) vinculados hacia el reequilibrio de las fuerzas en favor de los más débiles hoy amenazados. Las recientes decisiones tomadas en el marco de la Unión Europea ponen de manifiesto que dicha empresa no está llamada al fracaso.

Finalmente, se concluirá aquí una "atracción estratégica" del derecho francés. 
La perennización de su influencia es una prueba del mantenimiento de una diversidad cultural en un mundo marcado por múltiples juegos de influencias queridas o no, sufridas o aceptadas, que se ejercen hoy en día más que nunca sobre el derecho. Lo que está en juego es aún más fundamental, teniendo en cuenta que la época contemporánea se caracteriza por una influencia creciente del Derecho en las relaciones sociales, bajo una forma preventiva o curativa. Desde tal perspectiva, la defensa y la promoción del derecho francés y de otros sistemas jurídicos, responden a una expectativa importante de numerosos Estados, en particular, del Tercer Mundo, los cuales están preocupados por escapar a la aparición en el mundo de un derecho elaborado por el más potente de los potentes o esencialmente animado por las preocupaciones de la inversión y de las grandes empresas. Entendido así el derecho francés, es un actor de esa multipolaridad jurídica, cuyo concepto queda por integrar en los análisis de los partidarios de la multipolaridad política y cultural.

En otro sentido una característica constante del Derecho francés ino es la de tener la capacidad (rara) de soportar las influencias inevitables e innegables, para integrarlas a un corpus jurídico, sociopolítico e ideológico y elaborar nuevas especializaciones y síntesis? ¿ No es éste uno de los retos lanzados a los países del Tercer Mundo, en plena evolución, a menudo enfrentados a la dificultad de efectuar la puesta al día, jurídica y política, para enfrentar fuertes cambios socio-económicos, tradiciones y conservatismos firmemente afianzados? Al respecto, la doctrina de la tradición jurídica francesa, que existe más allá de las fronteras del hexágono, tiene ciertamente un papel que jugar: fuerte gracias a una probada metodología, fuerte también en valores que caracterizan históricamen- te el patrimonio jurídico francés, pero también, fuerte en las evoluciones que vuelven el derecho francés más atractivo.

Por último, en tal contexto y ante las relaciones de fuerza que sacuden el planeta, el destino del derecho francés está ligado al destino del derecho europeo y comunitario a cuya construcción contribuye, sin fundirse completamente con él. Allí como en otros lugares en un mundo, cuyo derecho global se construye, ineluctablemente, sea que se desee o que se lamente, retengamos que el derecho francés no ejercerá su influencia con sus innovaciones si él mismo no evoluciona y si no es capaz de evaluar sus propias debilidades y de ponerles remedio. Por otra parte, es lo que hace en proporciones considerables desde hace algunos años. El derecho francés conoce numerosos cambios, como prueba para tomar un ejemplo que nos es familiar, el derecho administrativo: en algunos años se volvió más sensible a las expectativas sociales o incluso empresariales contemporáneas; más receptivo al movimiento de subjetivización que caracteriza la época actual; más operativo en el mundo de los negocios. Las evoluciones más recientes de la jurisprudencia administrativa son ilustraciones de ello. A la vez, es a esta evaluación del derecho francés y del enfoque que tiene la doctrina francesa del derecho, así como a una reflexión sobre la competitividad de uno y otro, que éste coloquio nos pide expresarnos. Gracias a los organizadores por haber creado ésta ocasión para los franceses de reflexionar una vez más sobre su sistema que debe seguir evolucionando, pero también para los colombianos, cada vez más solicitados, algunos dirán tentados, de inspirarse en otras fuentes que las francesas y que han prevalecido hasta ahora. 